\title{
The Medical and Economic Roles of Pipeline Pharmacogenetics: Alzheimer's Disease as a Model of Efficacy and HLA-B*5701 as a Model of Safety
}

\author{
Allen D Roses ${ }^{*, 1}$ \\ ${ }^{1}$ Deane Drug Discovery Institute, Institute for Genomic Sciences and Policy, Department of Medicine (Neurology), Duke \\ University Medical Center, Cabernet Pharmaceuticals Inc., Fuqua School of Business, R David Thomas Executive Training \\ Center, One Science Drive, Suite 343, Durham, NC, USA
}

Pharmacogenetics (PGX) is the study of drug response as a function of an individual's DNA. PGX is often viewed as an extension of disease association genetics, and although this information may be related, it is not the study of drug response. Although medicines are used to treat diseases, the value of strategies that identify and incorporate DNA biomarkers associated with clinical efficacy, or DNA biomarkers for untoward clinical responses, can be applied directly to pharmaceutical pipelines. The growth of adverse event PGX studies involving marketed medicines generally uses relatively large numbers of affected patients, but has been productive. However, the two critical strategies for pipeline genetics must make use of fewer patients: (1) the early identification of efficacy signals so that they can be applied early in development for targeted therapies and (2) identification of safety signals that can subsequently be validated prospectively during development using the least number of patients with adverse responses. Assumptions are often made that large numbers of patients are necessary to recognize PGX hypotheses and to validate DNA biomarkers. In some ways, pipeline pharmacogenetics may be viewed as the opposite of current genome-wide scanning designs. The goal is to obtain PGX signals in as few patients as possible, and then validate PGX hypotheses for specificity and sensitivity as development trials go forward - not using hundreds of thousand of markers to detect strong linkage disequilibrium signals in thousands of patients and their controls. Drug development takes 5-7 years for a drug candidate to traverse to registration - and this is similar to the timeframe for validating genetic biomarkers using sequential clinical trials. Two important examples are discussed, the association of APOE genotypes to the demonstration of actionable efficacy signals for the use of rosiglitazone for Alzheimer's disease; and the identification of HLA-B*5701 as a highly sensitive and specific predictive marker for abacavir treated patients who will develop hypersensitivity syndrome (HSS). The rosiglitazone study prevented pipeline attrition by changing the interpretation of a critical Phase IIB proof of concept study (2005) from a failed study, to a positive efficacy response in a genetically predictable proportion of patients. Now, three years later, a Phase III program of clinical trials using pharmacogenetic designs is months away from completion (late08). If successfully registered (early09), millions of patients could benefit, and efficacy PGX would have achieved its first prospective block-buster. The use of safety candidate gene association genetics in patients who received abacavir therapy and developed HSS starting in 1998 culminated in a double blind clinical trial that determined sensitivity $>97 \%$ and specificity $>99 \%$ in 2007 . Clinical consensus panels rapidly recommended abacavir as the preferred therapy along with HLA-B*5701 pre-testing, immediately increasing the market share of abacavir with respect to other reverse transcriptases that are associated with there own adverse events. Targeting of medicines during drug development is now possible, practical, and profitable.

Neuropsychopharmacology Reviews (2009) 34, 6-17; doi: I0.I038/npp.2008.I53; published online 15 October 2008

Keywords: pharmacogenetics; Alzheimer's; efficacy; safety; pharmacovigilance; genome-wide association

${ }^{*}$ Correspondence: $\operatorname{Dr}$ AD Roses, Deane Drug Discovery Institute, Institute for Genomic Sciences and Policy, Duke University, Cabernet Pharmaceuticals Inc., Fuqua School of Business Incubator, R David Thomas Executive Training Center, One Science Drive, Suite 343, Durham, NC 27708, USA, Tel: +1919660 8065,

E-mail: roses@cabernetpharma.com

Received 20 July 2008; revised 15 August 2008; accepted 15 August 2008
The drug industry definition of pharmacogenomics is 'the study of variations of DNA and RNA characteristics as related to drug response,' and that of 'pharmacogenetics' (PGX) is the study of DNA characteristics as related to drug response. Internationally harmonized definitions were recently published as a 'Guidance for Industry' document by the US Food and Drug Administration (E15 definitions for genomic biomarkers, pharmacogenomics, pharmacogenetics, genomic data and sample coding categories, 2008). PGX can be applied to two fundamental clinical questions 
during drug development: (1) Are there predictors for drug candidate efficacy that can be validated in subsequent clinical trials? and (2) Are there predictors for side-effects, adverse events or metabolic differences that are associated with the use of the molecule in people? The first describes the potential enrichment of subpopulations of patients responsive to a drug; the second describes the risk of individuals for an adverse response.

PGX differs fundamentally from disease genetics based on the population at risk. For disease genetics, the population is open-ended, the PGX response only occurs in those individuals who receive the drug and not in anyone, patients or not, who has not been exposed to the drug (Roses, 2008c). It is, however, also possible to investigate disease genetics during clinical drug programs. PGX studies can determine associations related to the mechanism of disease clinical expression using patients enrolled in clinical trials to suggest additional targets for drug discovery. Disease mechanism genes have been frequently associated with PGX responses and therefore would also be included in candidate gene panels that are tested for PGX hypothesis generation. Candidate gene associations tailored to the knowledge or suspicion of a mechanism can also form either hypotheses for subsequent clinical trials, or provide a direction for specific target development. Thus disease associations and pathways can define PGX tools but also contribute to exploratory discovery.

During the 'pre-genomic' era before common genome-wide association screening [pre-2005], there were many instances where gene associations supported the involvement of disease mechanism candidate genes, or drug mechanism of action candidates. Hirschhorn et al (2002) reviewed reported gene associations with two or more replications and found that, although replication of reported associations was uncommon, when present it was frequently associated with genes related to the metabolism, binding or pathways. Goldstein et al (2003) similarly discussed the relevance of candidate genes related to the knowledge of the mechanism or pathway (Need et al, 2005; Roses, 1996, 2004).

'examples illustrate clearly defined groups of candidate genes that could harbor variation relevant to variable drug response: the first contains genes such as those that encode DMEs and drug transporters, which control the PHARMACOKINETIC properties (including DISPOSITION) of the drug; and the second group contains genes that encode drug targets (plus elements of the associated pathways) that influence drug PHARMACODYNAMICS. The second category includes not only the specific target of the drug, but also the broader pathway in which the target acts. To this we also add molecules that are similar to the target, which the drug might also modulate such as the effect that many non-cardiovascular drugs can have on potassium channels and thereby on QT-INTERVAL duration. One notable feature of pharmacogenetics is how often the obvious candidate genes carry variants that seem to influence drug response (Goldstein et al, 2003)'.

\section{GENOME-WIDE ASSOCIATION} INTERPRETATIONS - THE 'LINKAGE' OF THE $21^{\text {st }}$ CENTURY

In the last two decades of the 20th century, genetic linkage studies proved very useful to map and identify the mutations associated with classical Mendelian diseases, including autosomal dominant, autosomal recessive, Xlinked or mitochondrial genome disorders. These studies require the collection of family resources, including clinical data and DNA samples. Despite the difficulties of dealing with new forms of informed consents and clinical genetic counseling, particularly for normal family members at risk, linkage studies were incredibly successful in addressing genes for Mendelian-inherited diseases (McKusick, 2006, 2007). Of the thousands of clinical entities identified, most were extremely rare. Many common diseases were viewed as rather homogeneous and, in fact, prior to 1980 genetic etiologies were not strongly considered for cancers, Alzheimer's disease (AD), and many other common disorders (McKusick, 2006, 2007). The discovery of APOE4 as a major risk factor for 'sporadic' AD and the discussions of complex diseases that followed in the 1990s led to the rationale for supporting the Human Genome Projects and creating the tools for association studies and linkage disequilibrium (LD) mapping (Saunders and Roses, 1993; Strittmatter et al, 1993; Corder et al, 1993). Complex diseases have indeed been complex, and the failure to replicate the enormously statistical significance for APOE4 was rationalized by assertions of great genetic heterogeneity. However, failure to rapidly find many gene associations in complex diseases does not prove that they cannot be found by the application of other technologies. At this point in time, genome-wide association studies are identifying large numbers of genes with 'almost' statistically significant results (McCarthy et al, 2008). What are missing are newer methods to determine the effects of two or more associations: how to combine biology with genetics. A forerunner for this type of experimental strategy is provided in a short discussion of a second major interacting gene for $\mathrm{AD}$, summarized later in this communication. The use of densely genotyped candidate pathway genes reduces the number of statistical tests, provides specificity with limited number of patients, and is being "piloted" in pipeline safety PGX, and may soon be able to decipher the complex interactions of complex diseases (Martin et al, 2000; Zaks et al, 2006; Ahmadi et al, 2005; Roses, 1997).

The current limited experience with genome-wide SNP scanning using 500,000 or more than a million SNPs has created a fascinating situation. (McCarthy et al, 2008; Frayling, 2007; Zanke et al, 2007; Warren et al, 2007; Zeggini et al, 2007; Sladek et al, 2007; North et al, 2004; Libioulle et al, 2007; Wellcome Trust Case-Control Consortium, 2007; Raelson et al, 2007; Coon et al, 2007; Scott et al, 2007; Hunter et al, 2007; Schymick et al, 2007; Li et al, 2008). The time required to genotype and analyze the data derived from pre-existing clinical collections can be as little as 
several weeks. However, because of the large number of SNPs that need to be considered, the statistical corrections for most of these studies in complex diseases have resulted in only a few associated genes with more than borderline statistical significance. A greater number have borderline significance, which surely includes false-positive gene loci. Some may confirm independently previously performed genetic linkage and association studies (see Diabetes Mellitus below), but others may be expected to be falsepositives (Ioannidis, 2007).

Large well-characterized collections of several thousand patients and controls are now recognized as limiting factors, specifically assessing and accessing clinical phenotypes, the time and expense to identify and consent patients and controls, and the lack of availability of readily available populations for confirmation. The amount of work to collect, consent, examine and test thousands of patients is enormous and expensive-yet as data from additional diseases become available several unfortunate realities are becoming clear. The first is that most complex diseases, unlike the $\mathrm{AD}$ example, do not have extremely highly significant associations $(p \ll \mathrm{E}-8)$ after correction for the number of SNP tests $(5 \mathrm{E}+5$, or $6 \mathrm{E}$ ) (Coon et al, 2007; $\mathrm{Li}$ et al, 2008). The second reality is that there are usually $10-$ 100 genes identified with borderline significance suggesting that many large clinical series will need to be combined (Wellcome Trust Case Control Consortium, 2007). The third reality can be disappointing to many scientists with relatively fixed hypotheses - many widely accepted popular candidate genes are located within LD groups of genes that demonstrate no significant genome-wide association signal using genome-wide association scanning (Roses et al, 2007b; Meng et al, 2007; Dreses-Werringloer et al, 2008; Anandatheerthavarada et al, 2003).

In $\mathrm{AD}$ research, the amyloid precursor protein (APP) (metabolism and consequences) has been at the center of modern AD research, even earlier than the 1988 discovery of the APP gene and the subsequent genetic mutations associated with a rare early-onset form of the disease (Strittmatter et al, 1993; Schmechel et al, 1988; Bahmanyar et al, 1987; Goldgaber et al, 1987; Goate et al, 1991; St George-Hyslop et al, 1987; Tanzi et al, 1987; Citron et al, 1997; Sherrington et al, 1996; Kamino et al, 1996; Schellenberg et al, 1992). Current therapeutic agents related to amyloid are being investigated in early clinical trials but, to date, clinical benefit to patients has been elusive (Okura and Matsumoto, 2008; Pride et al, 2008). However, although the APOE gene is located within an LD region associated with an extraordinarily high significance, the APP gene does not appear to be associated with $\mathrm{AD}$ using genome-wide association scanning (Roses et al, 2007b). The so-called 'Amyloid Cascade' involving caspase enzymes may actually be a downstream consequence of mitochondrial toxicity (Townsend et al, 2007; Selkoe and Wolfe, 2007; Walsh and Selkoe, 2007; Dunys et al, 2007; Lee et al, 2007). APOE4 and APOE3 fragments without the C-terminal region may well be a form of pro-apoptotic death signals, often discussed in the oncology literature, with APP one of many catabolized molecules - but one which forms aggregates in tissue (Ziedan et al, 2008; Ashkenazi and Herbst, 2008; Hisatomi et al, 2008; Mahley et al, 2006; Roses et al, 2007a).

Unfortunately, there appears to be a relaxation of the rules of evidence for genes identified by genome-wide association (Dahlman et al, 2002). Formerly, because of the lack of validation observed with most reported association, when candidate gene papers were reviewed for publication in high quality journals, replication became a frequent requirement and biological validation studies were also included in the publication. Over several decades it had become clear from the literature that less than $5 \%$ of published disease association journal reports were ever confirmed and validated (Hirschhorn et al, 2002; Dahlman et al, 2002). McCarthy et al (2008) recently reported that more than 50 disease genes were found to be associated with a variety of diseases using genome-wide association scanning. This report "identified genes" which were not yet replicated, even between each of several genome-wide association studies of the same disease (see below). Very few met the candidate criteria adopted before genome-wide association scanning.

\section{EXAMPLE: TYPE 2 DIABETES MELLITUS (T2DM)}

The data from multiple T2DM genetic studies support the view that very large clinical case-control series are necessary to provide statistical significance for loci 'identified' by genome-wide association scanning. Increasing the density of coverage to over a million SNPs may, in fact, make it more difficult to identify statistically significant loci by needing to introduce a larger statistical correction. As an example, a published T2DM genome-wide association scanning was performed as part of the Wellcome Trust Collaborative Group study of seven diseases (Wellcome Trust Case Control Consortium, 2007). The analyses revealed three loci of significance - each barely below the corrected significance levels and nothing as dramatic as the APOE region of AD (Wellcome Trust Case Control Consortium, 2007). The association of the transcription factor 7-like gene (TCF7L2) illustrates the interplay between genome-wide association scanning and the previously more focused genetic association and linkage studies. In this case, genome-wide association scanning actually confirmed earlier gene linkage and association data. After reporting linkage of T2DM to chromosome 10q, Grant et al (2006) genotyped 228 microsatellite markers to Icelandic individuals with T2DM and controls over a $10.5-\mathrm{Mb}$ interval on $10 \mathrm{q}$ and reported linkage. Although it is clear that most reported linkage and targeted association results can result in many false-positives, these investigators found that a microsatellite variant within intron 3 of TCF7L2 was associated with T2DM, $(p=2.1 \mathrm{E}-9)$ (Hirschhorn et al, 2002; Helgason et al, 2007). This was then replicated in a US cohort, $(p=3.3 \mathrm{E}-9)$. These data were then confirmed by 
genome-wide association scanning containing $\sim 500 \mathrm{k}$ SNPs in 2000 patients (Wellcome Trust Case Control Consortium, 2007). In this case the gene linkage and gene association studies were performed prior to genome-wide association scanning, and TCF7L2 was confirmed ( $p=5.7 \mathrm{E}-13)$. Two other previously reported loci, FTO (chromosome 16) and CDKAL1 (chromosome 6) were also suggested by genomewide association scanning, but with more modest $p$-values.

Sladek et al (2007) also 'identified' four other loci containing variants that confer risk for T2DM, in addition to confirming the association with the TCF7L2 gene. These loci include "a non-synonymous polymorphism in the zinc transporter SLC30A8, which is expressed exclusively in insulin-producing $\beta$-cells, and two linkage disequilibrium blocks that contain genes potentially involved in $\beta$-cell development or function (IDE-KIF11-HHEX and EXT2ALX4)." (Sladek et al, 2007) Other than the TCF7L2 confirmation, the other three loci (on chromosomes 8, 10, and 11) were different than the second and third loci (chromosomes 6 an 16) in the Wellcome Trust study (Wellcome Trust Case Control Consortium, 2007). Followup dense mapping of these particular regions may find additional associated SNPs and add support to a locus of borderline significance (Zaks et al, 2006; Roses, 2008a). This example, however, illustrates the interplay between genomewide association scanning and more focused association studies. It may actually be more difficult than investigators imagined discovering new genes using primary genomewide association scanning data. After genome-wide association scanning, more focused secondary candidate analyses are needed in independently ascertained sets of patients to confirm borderline significant SNPs or regions.

\section{EXAMPLE: WHERE DOES AD DISEASE GENETICS END AND PGX BEGIN?}

Genome-wide association scanning data from studies of $\mathrm{AD}$ patients have unequivocally identified the $\mathrm{LD}$ region that contain the apolipoprotein E gene (Coon et al, 2007; Li et al, 2008). One variant, APOE4, has been widely replicated as a confirmed susceptibility gene since the initial publications in 1993 (Saunders and Roses, 1993; Strittmatter et al, 1993; Corder et al, 1993). However, the genome-wide association scanning data resulted in a remarkable 'coincidence' observed in cell biology studies involving the co-localization of APOE and tomm40 proteins to the outer mitochondrial membrane. This interacting gene, TOMM40 (the outer mitochondrial membrane channel gene) was first encountered during 1997 genetic epidemiology studies modeling whether LD mapping around APOE would have found the gene faster than the years it took 5 years earlier in 1992 (Lai et al, 1998). The then-unknown genetic polymorphisms, called PEREC, were located adjacent to APOE within a small LD region. APOE co-localizes to the outer mitochondrial membrane, suggesting isoform-specific interactions leading to a potential role for APOE-induced mitochondrial apoptosis as an early step in $\mathrm{AD}$ expression (Chang et al,
2005). Biological data have demonstrated that the proportion of mobile mitochondria in neuronal cell culture, as well as the speed at which they move and the distance that they traverse, lead to increased mitochondrial apoptosis (Mahley et al, 2006; Roses et al, 2007a; Xu et al, 2006; Harris et al, 2003; Brodbeck et al, 2008). Phylogenetic data suggesting an independent genetic effect on the development of $\mathrm{AD}$ for TOMM40 are outlined below. The cis-acting event of a thenunknown protein in 1998 produced the Alzheimer-like pathology in APOE knockout mice that were transfected with human genomic DNA cosmid fragments (Roses et al, 1998). This was before the identification of TOMM40, or its adjacent location within an LD region.

The interaction between multiple haplotypes involving TOMM40 variants and APOE isoforms highlight an independent contribution of APOE3 to AD pathogenesis (Roses, 2008a, c; Li et al, 2008). Several of the TOMM40 gene variants evolved only cis-linked on APOE3 cistrons. Thus any added genetic effect of the TOMM40 variants segregates independently from APOE4 cistrons. This 'coincidence' of adjacent interacting proteins may account for the extraordinarily significant statistical association data found in all $\mathrm{AD}$ genome-wide association scanning studies. It is of interest to note that the initial commercially available genome-wide association scanning platforms did not contain any APOE polymorphisms but were identified with TOMM40 and APOC1 SNPs - but the region is virtually always referred to as the 'APOE' region. Perhaps the APOE and TOMM40 region would be more accurate with respect to $\mathrm{AD}$ pathogenesis.

These data, which combine disease genetics and putative molecular mechanisms of pathogenesis, can also be viewed within a clinical PGX context. Because of the strong genetic effect of inheriting an APOE4 allele, APOE4 has been referred to as a complex susceptibility gene for almost two decades. Consistent replications of the age of onset distributions as a function of APOE genotype confirm that the role of APOE3 inheritance is not totally benign, but is a lower risk factor observed at a slower rate of disease onset (Corder et al, 1993; Li et al, 2008). There are genetic variants of TOMM40 that are located only on DNA strands containing APOE3 in the LD regions (Roses et al, unpublished data), and thus not in Hardy-Weinberg's equilibrium as was required for SNPs qualifying for inclusion on genome-wide association panels. Phylogenetic changes in TOMM40 sequences that are cis-linked only to APOE3 can act to increase the risk of $\mathrm{AD}$ when they are on the same cistron as APOE3, whereas other variants of TOMM40 cislinked to APOE3 decrease the risk associated with APOE3. An independent genetic test is to determine whether those TOMM40 polymorphisms associated with less risk of $\mathrm{AD}$ segregate at a later age in onset of disease distribution plots for AD patients with APOE3-containing genotypes (APOE33 or APOE4-3), (Roses, unpublished data).

From the rosiglitazone clinical trial program detailed below, it has been possible to collect APOE allele-specific patient groups (APOE4-negative and APOE4-positive) to 
further confirm published age of onset data in a set of patients specifically encountered in a clinical trial setting. From the collections of DNA from treated patients in the trials, as well as patients not treated with rosiglitazone, it will be possible to independently treat the putative predictions emerging from the study of TOMM40 variants linked to APOE3 and not APOE4 or APOE2. Thus there is interplay between statistical genetics and mechanisms of disease - as well as drug mechanisms - that may be more clearly illustrated following the conclusion and analyses of the rosiglitazone AD clinical trials in 2009.

\section{EXAMPLE: THE HISTORY OF THE MECHANICS OF AN AD CLINICAL TRIAL WITH AN EFFICACY PGX PROSPECTIVE HYPOTHESIS}

The most important point along the drug discovery and development pipeline is the demonstration of proof of concept for efficacy (POC). There are really two factors that determine whether a drug candidate is deemed effective. If the POC is prospectively predicted in the protocol for a clinical trial, then the regulators consider the trial as hypothesis testing. But if the results (clinical or PGX) are determined post hoc, then the data are considered exploratory and must be tested prospectively in subsequent trials. The difference between two sequential positive hypothesis testing trials and three longitudinal conformational registration trials can extend the development program by several years. Clinical trials frequently take many months or several years to set up and complete. The importance of testing prospective hypotheses in the earliest stages of drug development (especially in terms of the always-expiring market exclusivity) is a major commercial factor. Even if 200 prospective hypotheses are simultaneously tested in the initial Phase IIA trial, and only one seems to predict efficacy, then the developer may be one step closer to confirmation and possible drug registration (Roses et al, 2007a).

Rosiglitazone for the treatment of $\mathrm{AD}$ provides a clear demonstration of a failed study in the intention-to-treat (ITT) patients. Like many such studies, this program would have been terminated and efficiently dropped from the portfolio in less than a week after results became available. However, because there was a prospectively defined hypothesis - in this case, that APOE4 carrying AD patients would respond less well than $\mathrm{AD}$ patients who carried no APOE4 alleles, efficacy for APOE4 non-carriers was defined within a week following the original ITT analyses. It is important to understand the timing for pipeline PGX. Clinical data become available only after the conclusion of trial and the phenotypic data define the efficacy analyses of the genetic hypotheses. Efficacy PGX analyses can only commence following the identification of those patients who respond with clinical end points of efficacy. Even in this case, it took an intervention by the Chair of R\&D to hold back the efficient killing of a program even for 1-2 weeks to complete the PGX analyses. Attrition can be habit forming and insanely efficient.

In this case, within a week after the patients who responded with efficacy were identified, a totally 'failed' experiment was converted to a successful efficacy PGX result for which the clinically responding population had been predicted by the prospective hypothesis. This was a textbook case of preventing attrition, and the effect that efficacy PGX can have on the critical POC step in drug development. In $\mathrm{AD}$, the clinical end points for efficacy accepted by the regulators had been met by approximately half of the ITT patients - but a predictable half of a huge population with a severe, unmet medical need.

Phase III involved recruiting and following thousands of patients for 48-week clinical trials. These trials, and their PGX designs, must be pre-planned to be efficiently initiated and mobilized. In the most efficient circumstances of pipeline pre-planning, working with the regulators with respect to the End of Phase II meeting, establishing the new clinical centers, and recruiting patients can take a year or more to get off the ground. The clinical trial actually starts the day that the first patient enters the study and ends the day that the last patient completes the study. The first two studies began in 2005 and will close before the end of 2008 . The jury is still out (currently August 2008) but a verdict is expected in early 2009.

Figure 1 illustrates the Phase II data (Risner et al, 2006). On the left side are the results of treatment with three different doses of rosiglitazone monotherapy over a 24week study. Under any conditions of interpretation, there is absolutely no evidence that there was any efficacy in any treated group of patients in this study, nor in the placebo group - a dismal failure. These were the initial data available at the end of the study.

However, after PGX analyses and a week delayed, there was genotype-related efficacy for only those patients who did not carry an APOE4 allele (approximately 50\%). Each of the groups receiving one of the three doses illustrated a measurable improvement on the Alzheimer's disease Assessment Scale for cognition (ADAS-cog scale, accepted as a primary end point by the regulatory authorities), as well as secondary end points. The combined placebo group at all three doses for all APOE4-negative AD patients did not improve but demonstrated slight deterioration over the 24 weeks of the Phase IIB monotherapy study. The difference between the placebo and the drug met the three point criteria' expected by the FDA for the ITT group who carried no APOE4 allele. Of course, as would be predicted from the whole ITT population data, carriers of one or two APOE4 alleles showed no improvement.

An important new process in the FDA, the Voluntary Genomic Data Submission, allowed a non-decisional discussion with the FDA leadership regarding the interpretation of the PGX data, and proposals for the Phase III registration programs (Orr et al, 2007; Lesko, 2007). A spirited VGDS discussion allowed a transparent debate to 

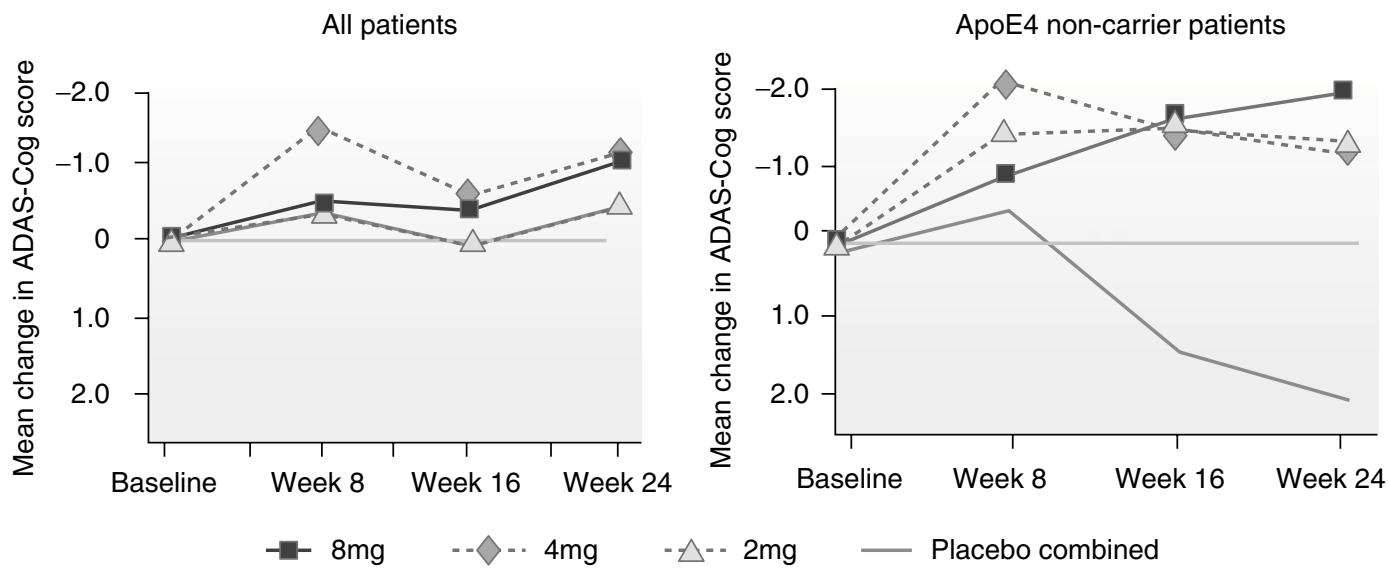

Figure 1. The left panel represents the ADAS-cog results for all patients who were treated with rosiglitazone with any of the three doses versus their controls. Negative values are associated with clinical improvement, with an effect of approximately $-3 \cup$ when compared with placebo-treated patients is considered actionable for consideration for a phase III registration program. There was no evidence of efficacy. The right panel illustrates the effect of three different doses of rosiglitazone for all treated patients who did not carry an APOE4 allele [APOE3/3 or APOE2/2 genotypes]. The prospective hypothesis in the study protocol was that APOE4 carriers would not respond to rosiglitazone as well when compared to non-carriers. [see text] The combined patients who received placebo are also illustrated, and a positive clinical improvement is observed. Thus, the PGX hypothesis appeared to be confirmed in a proof-of-concept phase IIB study.

occur - most of which involved the views of various experts within the FDA. A consensus view of the possible designs of a Phase III program allowed the End of Phase II Meeting (held the following month) to subsequently proceed efficiently. An APOE-specific clinical trial design, which would evaluate APOE4-negative and APOE4-positive patients was adopted. By recruiting patients with each of the four common genotypes, APOE3/3, APOE $4 / 4, \mathrm{APOE} 3 / 4$ and APOE2/3 (only a few APOE2/4 and APOE2/2 individuals will be recruited) genotype-specific analyses will be available. Two different doses of rosiglitazone were used with the low dose equivalent to $25 \%$ of the commonly used daily dose for the treatment of T2DM. Of particular interest, besides efficacy, in these large studies is whether there will be any genotype-specific side-effects or adverse events (AEs). One important fact that is certainly diagnosable before the study concludes is that there is no increased frequency of myocardial infarction deaths in any of the three phase III populations to date. This issue was raised inappropriately for T2DM treatment based on a flawed statistical meta-analysis assuming all cells of data were equivalent and using a US Congressional government hearing to damage the reputation of rosiglitazone, resulting in a delay of recruitment late in the course of the $\mathrm{AD}$ trials (Nissen and Wolski, 2007; Goldberg, 2007; Fovenyi, 2006; Gerstein et al, 2004, 2006; Lago et al, 2007).

\section{TWO MORE RECENT CLINICAL TRIALS REPORT IMPROVEMENT IN APOE4 NON- CARRIERS: ANOTHER COINCIDENCE?}

There have been two press reports involving two quite different therapeutic agents that associate an efficacy response in treated $\mathrm{AD}$ patients to the presence or absence of an APOE4 allele. Each is leading to a PGX design that will be used in additional clinical trials. If these proposed treatments are approved in subsequent years, then the possibilities for multiple-drug therapies affecting pharmacogenetic genetic subgroups of patients may become a reality. In fact, if rosiglitazone is approved in 2009, it may become incumbent upon follow-on drug development programs to demonstrate non-inferiority, or even complementary or additive effects.

Accera, a small biotechnology company, announced its plan "to commercialize a first-in-class medical food product to treat Alzheimer's disease, called Ketasyn, which is particularly effective in APOE4-negative patients." (http:// www.pgxreporter.com/issues/5_43/features/143237-1.html).

Similarly, recently announced results from Phase II clinical trials for the investigational Alzheimer's drug bapineuzumab (an antibody to clear amyloid $\beta$-peptide) shows the drug may be beneficial in patients who are noncarriers of the APOE4 allele. It was also mentioned that there may be a higher incidence of vagal edema in APOE4positive patients. (http://www.pgxreporter.com/issues/6_27/ features/147947-1.html). Whether these initial reports are validated, the fact remains that allele-specific APOE subsets of patients have been defined by apparent clinical responses - that will be tested in subsequent clinical trials.

From a PGX point of view, it is interesting to note that both developers of other early $\mathrm{AD}$ treatment programs performed post hoc, retrospective PGX evaluations of their data using the inheritance of an APOE4 allele, and have reported targeted 'successes.' Both of these drug candidates have yet to enter Phase III trials, and further data supporting the press reports are not yet available. However, because neither company had proposed the PGX hypothesis 
prospectively, each study can only be considered as 'hypothesis generating,' nor could either trial be used as one of the two positive studies required for drug registration, that is, two more studies would required at a minimum. In this case, Ketasyn will probably be tested as a medical food.

\section{A COMMENT ON THE TENSION BETWEEN AN R\&D VIEW AND A COMMERCIAL VIEW}

If rosiglitazone is approved and reimbursed in 2009, the commercial view of efficacy PGX will change rapidlymany years after the R\&D strategy was conceived and executed. The same industry analysts who discount the chance of success in 2008 may be the 'earliest' proponents of pipeline PGX in 2009. Nothing will have changed in PGX science - only the perception of those who control the R\&D budgets within the pharmaceutical industry. PGX predictive science is evaluated prospectively, using experimental strategies that took many years to pilot. Economic success is always evaluated by the 'here and now'.

\section{OTHER NEUROPSYCHIATRIC AND COMPLEX CLINICAL APPLICATIONS OF PIPELINE PGX}

In the context of neuropsychopharmacology there are some special difficulties in evaluating clinical trials. Using the historic paradigm of determining the difference of an average response in defined clinical end points with the active drug candidate or placebo, it has been extremely difficult to generate consistent efficacy with many newer agents (Malhotra et al, 2004). This raises the question of whether the efficacy responses observed with some of the older drugs are appropriate for different mechanisms of action of newer drug candidates. Most clinical trial designs were based on historic assumptions regarding the homogeneity of neurological and psychiatric diseases. This may have contributed to defining end points for studies that only powerful drugs could meet, but unfortunately are also associated with very significant side-effects and adverse events (AEs). On the other hand, experience with several decades of psychiatric treatment has created a knowledge base for PGX-based candidate gene panels derived from those mechanisms.

Serretti et al (2008) have recently reviewed PGX studies in depression and proposed methodological guidelines not only including end points but also sampling, diagnostic criteria, compliance and trial designs. There have been many PGX studies in mood disorders "after the initial reports linking gene variants to treatment outcomes. However, a considerable range of methodologies has been used, making it difficult to compare results across studies.... Specification of sampling source (inpatients vs outpatients, primary vs tertiary settings), standardization of diagnostic systems and treatments, adequate monitoring of compliance through plasma levels, sufficient length of observation (at least 6 weeks for acute antidepressant treatments, though 3-6 months are preferable), the use of a range of response criteria and the inclusion of possible environmental confounding variables (life events, social support, temperament) are all potentially important issues when planning pharmacogenetic studies." This scholarly review presents suggestions and recommendations for approaching confirmation of depression studies and other mood disorders.

Oncology and virology are therapeutic areas that have led the way in determining the PGX responses to drugs, in no small part aided by the knowledge of basic mechanisms and the ability to get drugs into the marketplace faster because of accelerated marketing programs in the United States, and conditional approvals in the EU (Anonymous, 1992). It is interesting to contrast this situation with the extreme heterogeneity of inherited psychiatric and neurological diseases. DNA variants in many different genes in the genome frequently define virtually identical phenotypes in disease categories that had previously been considered under one clinical diagnosis, such as the hereditary spinocerebellar diseases, hereditary spastic parapareses and others (Jani-Acsadi et al, 2008; Russman, 2007; Koeppen, 2005; Passamonti et al, 2004). There are now tens of specific and diverse mutations, which define various endo-phenotypes and inheritance patterns. It is unclear whether, even in these genetically classified disease groups, similar treatment will be successful. Therefore, why should we assume that complex neuropsychiatric diseases are different simply because we still have difficulty in identifying the contributing genes, and measure an 'average response' to drug therapy across heterogeneous patients $v s$ placebo? Perhaps this is a clue that complex diseases like schizophrenia and depression are 'complex' and heterogeneic perhaps not.

Diseases have often been defined by drug-responsiveness before PGX entered the lexicon. Steroid-responsive asthma was an example of category of description over many decades without knowledge of mutations, polymorphisms, or the basis of steroid-responsive heterogeneity. Depression, schizophrenia, Alzheimer's dementia, obesity, heart diseases, and other complex neuropsychiatric 'diseases' are frequently subclassified by pathology or drug-responsiveness - with little idea whether there are many etiologies and certainly no methods of prediction. One might assume that these 'diseases' may well become drug-defined when efficacy PGX allows a better prediction of responsive phenotypes. To better understand the role of PGX in drug development, pharmacovigilance and future medical care, some recent examples will be presented and briefly discussed.

\section{SAFETY PGX — FOR THE DEVELOPERS IT IS ALL ABOUT TIMING}

The list of clinical trials in depression that have had one successful trial and multiple efficacy failures is long. 
The trials are dependent on all the factors mentioned above, as well as two over-arching facts: (1) the clinical definitions used in the studies, even if internally consistent, may still result in the collection of a different spectrum of clinical heterogeneity in subsequent studies, even with the same drug and the same physicians; and (2) failure to reproduce the successful trial design using PGX data. Similar to these data presented above for APOE alleles and $\mathrm{AD}$, it may be possible to define more accurate and responsive patient selection using genetic markers and PGX trial designs (Roses, 2008b).

Adverse events are not specifically related to only neuropsychiatric drugs, but represent a common problem for all drugs. When the end points of a trial are defined clinically, as in many neuropsychiatric studies, a stronger drug is generally needed to significantly affect end points during the timeframe of the trial. Small subtle effects are difficult to quantify and may take longer to be appreciated by current clinical end point measurements. Stronger drugs and higher doses amplify the appearance of adverse events.

From the drug developer's point of view, the management of adverse events is all about timing. When uncommon, but not rare, side-effects or adverse events are noted during development, efforts should be made to understand any genetic contribution to the susceptibility for experiencing the adverse event - not trying to ignore its existence or using speculative rationales to put it aside. The difference is the ability to develop diagnostic biomarkers using PGX strategies. The clearest example in the literature is abacavir, a non nucleoside reverse transcriptase that is used to treat HIV infection. Approximately 5\% of patients who started the drug can experience a hypersensitivity syndrome (HSS) consisting of fever, rash, gastrointestinal symptoms and constitutional symptoms. The onset of most HSS occurs within 6 weeks of initiating abacavir in naive patients and can be managed by stopping abacavir therapy for alternative drugs now in the marketplace. Re-treatment of HSS patients with abacavir can lead to anaphylactic shock and death (Hetherington et al, 2001).

In 1997 abacavir was the first of its class - for what was then an almost invariably fatal disease. The regulators approved an accelerated marketing program for treatments of cancer and HIV, which allowed potentially life-saving drugs to be available to patients, but under a strict set of enforced risk management procedures. Included in the accelerated approval for abacavir was the contract to develop a predictive diagnostic marker to avoid dosing susceptible patients. A detailed historic review of the chronology of a diagnostic test for abacavir-induced HSS was recently published (Hughes et al, 2008).

From a public health point of view, acknowledging that all drugs have side-effects, accelerated approval allows patients to receive the benefit early in development and essentially mandated a risk management program that included active surveillance by the sponsor-not just voluntary reporting to a central database. The advantage to the patients is that the significant part of the revenue of the marketed drug must be devoted to enhancing safety. With regard to the industry, when adverse events and serious side-effects arise post-marketing for some drugs, the regulators can only use blunt tools for enforcement: either black-box warnings added to the drug label, or withdrawal of the drug from the marketplace. Over the past few years some very high profile drugs have been withdrawn from marketing and suffered serious legal and financial penalties - besides having already spent the entire costs of development, launch and marketing (Peck, 2007). The economics of safety defines the solution- learn what you can as early as possible and develop risk management plans that can define diagnostic tests.

Well before the genome was declared to be sequenced, two groups acting independently reported retrospective studies of HSS cases demonstrated a highly significant percentage of the HSS patients carried HLA-B ${ }^{\star} 5701$ (Hetherington et al, 2002; Mallal et al, 2002). GSK sponsored a large prospective clinical trial design to measure the value of pre-screening HIV patients with HLA-B ${ }^{\star} 5701$ and the frequency of HSS. In patch tested immunologically confirmed patients, this result was dramatic with both the specificity and the sensitivity of the test exceeding $97 \%$. None of the screened group developed HSS, whereas approximately $3 \%$ of the non-screened group who tested positive for the marker developed HSS (Mallal, 2007, 2008).

The economic effect is real. Abacavir is now the preferred treatment for initiation of therapy in naive patients, with the recommendation that all patients should be screened before initiation of treatment (Phillips and Mallal, 2008; Adult and adolescent guidelines HIV [abacavir], 2008). The economic consequences for the company (and the benefits to patients) reflect the fact that the drug has enormously increased its benefit to risk ratio. From a company perspective, the increase in revenue over previous projections in 1 year paid for the entire risk management program many times over. It is the first clear example based on the gold standard-a prospective double-blinded clinical trial of an adverse event, but it is certainly not a special case, and will be repeated many times over as regulators require and enforce better surveillance and risk management programs in the future (Mallal, 2008).

Everyone wins with safety. Not being proactive about safety by using existing PGX tools can only put a drug at risk. In fact, most drugs are not approved as accelerated marketing but become available to physician use only after launch. This creates a nightmare situation for drug companies - every academic investigator with access to treated patients can rapidly investigate safety signals whose only effect for the pharmaceutical company is negative. Why not protect the drug proactively and relatively inexpensively? Patients are safer, payers are more likely to reimburse, regulators are satisfied, and the asset is in an improved position in the marketplace. For companies with a reputation for embracing safety, the added gains of increased stock ownership and value will also be a factor defining success. 


\section{PGX AS A BUSINESS DRIVER}

The most important factor in drug discovery and development is reducing attrition, or failure once an asset has had a positive POC and is in the expensive phases of full development. The extreme negative case would be a fully developed molecule with additional Phase IV programs, which is removed from the market because of severe 'postmarketing' adverse events after total investment had been committed (Maron and Hauser, 2007; Epstein, 2007). In an industry where successes have been defined as a drug with a $\$ 1$ billion per year market, a few successes can sustain an otherwise productive company. However, included in the current huge estimates for cost of drug development are the costs of each failure (Reichert, 2003). Over the past decade estimates of drug costs have continued to increase because of the continuing attrition. When research and development are measured as a necessary expense, rather than the lifeline of a pharmaceutical company, the short-term economic perspective can impede productivity. Although the literature is full of high profile drugs that failed because of safety concerns, the pharmaceutical industry has been slow to protect its pipelines by the identification of those individuals at the greatest risk of adverse effects - and to use that information to increase the benefit to risk ratio. The mantra that a safety test would cut into sales is hollow, especially if the result is no sales (accompanied by expensive litigation) because of safety issues in full development or in postmarketing pharmacovigilance. Regulatory emphasis on evidence-based surveillance systems will increase the role of PGX to discover markers that predict the occurrence of an $\mathrm{AE}$ or side-effect that decreases the attractiveness of a safe and effective medicine. Yet the pharmaceutical industry continues to repeat failing procedures and to re-organize 10 to 12-year pipeline processes every 5-7 years. Albert Einstein, who was a reasonably perceptive individual said: 'Insanity is doing the same thing over and over again and expecting different results' (www.brainyquote.com/quotes/ quotes/a/alberteins133991.html). Yet increasingly obsolete perceptions within the financial and pharmaceutical communities ignore the chance to target medicines for safety and efficacy with existing PGX technologies.

Safety concerns are the main purview of regulators. Once some degree of efficacy is demonstrated, targeting patients for efficacy is actually a major concern for payers, both private and public. Reimbursement of an approved drug is the true measure of success. Registration of a drug with a 'we can sell it' strategy is becoming harder to sustain. The regulators defining safety more rigorously, but the payers control the marketplace. Targeting efficacy and safety through large PGX studies in health maintenance organizations or in publicly supported health systems will become far more important in the USA over the next decade-just as it already has become in European, Asian, and Canadian systems over the past decade. For pharmaceutical companies, the time to learn the most about your drug is during development and surveillance. Presenting a medicine that has a greater than $90 \%$ chance of a successful therapeutic response in a smaller targeted population is of far greater value than trying to continue to target all patients - because the target value is obtainable and not a cherished relic of the past. The best reason to expect return on investment is that payers will be more likely to reimburse efficacy for the targeted population, and not purchase innovative new medications for those individuals with a low risk of success.

\section{THE COMMERCIAL FOLKLORE OF SEGMENTING PATIENTS $v s$ REALITIES}

Why would a drug developer target therapy to only $30-40 \%$ of the patients? The tacit commercial assumption is that the drug would actually achieve $100 \%$ market share. Yet if real drugs are evaluated by market share, the assumptions about target populations are often woefully unmet. For example, one drug in the GSK portfolio which had $>\$ 1$ billion peak sales, achieved up to $14 \%$ of the market share at its peak sales. If the $40 \%$ of responsive individuals could be accurately identified using PGX tools during development, peak sales equivalent to more than three times the achieved market share could have been targeted much earlier during the commercial life of this drug. The issue of efficacy is in no way diminished when a drug becomes generic, and then there is even less incentive for research investment.

From an economic point of view, discovering and developing innovative safe and effective drugs is the first rule for success but, the real opportunities for companies to succeed also must include satisfying the "ethics" of reimbursement. New drugs that are effective will be reimbursed by HMOs, government agencies, and other payers. They (we) simply do not want to purchase drugs that are not predicted to work - nor do patients want to risk side effects and AEs with drugs from which they do not anticipate efficacy. A multiple-drug market with coverage for all responsive patients may well be the future of pharmaceutical medicine. To anticipate the future, pharmaceutical companies require up to a decade lead time for early discovery projects.

\section{PRACTICAL CONSIDERATIONS FOR DRUG DEVELOPERS}

Both of the examples (APOE and HLA-B ${ }^{\star 5701)}$ discussed in this review were applied as 'known' PGX markers. Both were discovered before they were applied to pipeline PGX, but were also confirmed as associations during clinical trials (Saunders and Roses, 1993; Corder et al, 1993; Hetherington et al, 2002; Mallal et al, 2002). Thus the role of pipeline PGX can also be viewed as having a drug discovery component, especially identifying associations of candidate genes and pathways, which could then be expanded to target selection. How would a drug developer apply this to the pipeline, especially for neuropsychiatric diseases? 
There are several important basic requirements for successful application of pipeline PGX. The first two are obvious and necessary: (1) Appropriate informed consent must be obtained and documented, and (2) Patients must have DNA collected and stored appropriately for regulatory submission. PGX should (3) start with the earliest clinical studies, for safety signals during Phase I, and to generate hypotheses early in phase IIA. The impact of starting with a smaller hypothesis generating trial allows hypothesis testing (as a registerable study) in Phase IIb. The APOE example was a Phase IIb clinical trial that demonstrated not only extensive hypothesis testing of other biomarkers than APOE alleles, but resulted in the customized, genotype-specific PGX Phase III Program design agreed with the regulators (Roses et al, 2007a; Risner et al, 2006).

Drug discovery is usually based on mechanistic hypothesis. If an exploratory discovery of an association of an SNP is real, it can widen the range of candidate genes and pathways and be associated with efficacy in early Phase II clinical trials. It is possible to test polymorphisms from genes related to all of the proposed hypothesis, as well as every other hypothesis that is discussed in the literature using customized arrays without substantially increasing the time or cost of the analyses by adding SNPs. If only one of $\sim 500$ genetic variants is associated, it provides a hypothesis and an expanded pathway that can generate additional candidate genes for the subsequent clinical trials. In addition, SNPs in LD with the associated variant can be used to confirm this region of the chromosome and possibly suggest new genes (Li et al, 2008). (4) This pipeline strategy is based on extensive, creative candidate gene lists, not initial genome-wide screening. In this manner statistical significance is corrected for $\sim 500$ tests, rather than 500000 or 1 million tests. Besides, if there is a known linkage association, genome wide scanning can be used locally, with the proposed analyses limited to the chromosomal region of linkage, thus substantially reducing the need for a correction based on the whole genome (Lai et al, 1998).

Predictive exploratory discovery can be performed with candidate genes. The most critical difference between pipeline PGX trials and academic studies of disease-related genes is the fact that the gene variants will be studied in a relatively smaller group of well-defined group of patients (Roses, 2008c). The difference is that only the patients who receive the drug can be classified as experiencing efficacy, or not. This is independent of disease gene studies, but may well eventually be demonstrated to provide a pathway definable subset of disease heterogeneity. The large number of patients required for genome-wide associations contrasts significantly with the modest number of patients in a Phase II trial. In addition, there is also the general assurance that if patients respond positively as defined by clinical end points, there will be a subsequent clinical trial with which to test any positive data derived from the first study. In the context of drug registration, successive clinical trials are designed to confirm positive signals and rule out false-positives (Mallal, 2008; Roses, 2007). Storage of DNA allows genome-wide analyses for additional markers for companion diagnostics once there are thousands of patients enrolled (Roses et al, $2007 \mathrm{~b})$. From the viewpoint of regulators, the initial 'discovery' of a new association for PGX is exploratory-or 'hypothesis generating.' If that candidate is identified and tested in subsequent clinical trials, that is considered 'hypothesis testing.' And, if the trial demonstrates no patients who improved relative to clinically defined end points, then there is no basis for another clinical trial or PGX analysis. The cost of pipeline PGX is relatively trivial when compared with the cost of the clinical trials - and the costs of drug attrition. PGX does not define the patients: but, rather serves to define those patients who responded with prospectively defined clinical end points. Sequential clinical trials can add to the significance of real associations, or rule out false-positives.

The opportunity in neuropsychiatry is especially large because PGX candidate analysis provides an opportunity to segregate drug responders who may give a mechanistic clue to otherwise obscure phenotypic and genotypic heterogeneity. The use of creative candidate lists that include any and all proposed disease gene hypotheses can serve to identify new mechanistic pathways - as had been the case for Alzheimer's disease, or the molecular basis of abacavir hypersensitivity. With modern genetic tools, derived from the genome, it is now possible to propose peer-defined 'outrageous' hypotheses - at little additional financial or statistical cost. Confirmation of such a hypotheses may also define a new pathway of drug discovery, but also qualify the next clinical trial for hypothesis testing regulatory interpretations and save valuable patent or exclusivity time.

\section{CONFLICT OF INTEREST}

Dr Roses is currently the Director of the Deane Drug Discovery Institute at Duke University which involves consulting with investigators regarding pathways to the discovery of new drugs. For consultative activities involving pipeline pharmacogenetics he has created Cabernet Pharmaceuticals Inc., an S-type (individual) corporation in the State of North Carolina, solely owned by Dr Roses and consulting associates. Cabernet Pharmaceuticals Inc., is a PGX consulting and project management company whose customers are pharmaceutical and biotechnical companies with pharmaceutical pipelines. Dr Roses was formerly Senior Vice President of Genetics Research and Pharmacogenetics in GlaxoSmithKline and a member of the Research and Development Executive Committee for 10 years. Dr Roses was a member of the FDA Science Board from 2003 to 2007 and currently consults for the FDA (gratis). Dr Roses has no affiliation with GSK, but owns GSK stock options, all of which expire in October 2009. In addition, Dr Roses owns stock in his GSK retirement fund worth more than $\$ 50000$, which is being liquidated over the 5 -year period after his retirement in October 2007. He has no other stock holdings greater than $\$ 50000$. He is on the Board of Directors of the American Dance Festival and is a Lifetime Iron Duke. 


\section{REFERENCES}

Adult and adolescent guidelines HIV [abacavir] (2008). http://www.Aidsinfo.Nih. Gov/contentfiles/adultandadolescentgl.Pdf.

Ahmadi KR, Weale ME, Xue ZY, Soranzo N, Yarnall DP, Briley JD et al (2005). A single-nucleotide polymorphism tagging set for human drug metabolism and transport. (see comment). Nat Genet 37: 84-89.

Anandatheerthavarada HK, Biswas G, Robin MA, Avadhani NG (2003). Mitochondrial targeting and a novel transmembrane arrest of Alzheimer's amyloid precursor protein impairs mitochondrial function in neuronal cells. $J$ Cell Biol 161: 41-54.

Anonymous (1992). New drug, antibiotic, and biological drug product regulations; accelerated approval — fda. Final rule. Fed Regist 57: 58942-58960.

Ashkenazi A, Herbst RS (2008). To kill a tumor cell: the potential of proapoptotic receptor agonists. J Clin Invest 118: 1979-1990.

Bahmanyar S, Higgins GA, Goldgaber D, Lewis DA, Morrison JH, Wilson MC et al (1987). Localization of amyloid beta protein messenger rna in brains from patients with Alzheimer's disease. Science 237: 77-80.

Brodbeck J, Balestra ME, Saunders AM, Roses AD, Mahley RW, Huang Y et al (2008). Rosiglitazone increases dendritic spine density and rescues spine loss caused by apolipoprotein e4 in primary cortical neurons. Proc Natl Acad Sci USA 105: 1343-1346.

Chang S, ran Ma T, Miranda RD, Balestra ME, Mahley RW, Huang Y et al (2005). Lipid- and receptor-binding regions of apolipoprotein e4 fragments act in concert to cause mitochondrial dysfunction and neurotoxicity. Proc Natl Acad Sci USA 102: 18694-18699.

Citron M, Westaway D, Xia W, Carlson G, Diehl T, Levesque G et al (1997). Mutant presenilins of Alzheimer's disease increase production of 42-residue amyloid beta-protein in both transfected cells and transgenic mice. [see comment]. Nat Med 3: 67-72.

Coon KD, Myers AJ, Craig DW, Webster JA, Pearson JV, Lince DH et al (2007). A high-density whole-genome association study reveals that apoe is the major susceptibility gene for sporadic late-onset Alzheimer's disease. [see comment]. J Clin Psychiatry 68: 613-618.

Corder EH, Saunders AM, Strittmatter WJ, Schmechel DE, Gaskell PC, Small GW et al (1993). Gene dose of apolipoprotein e type 4 allele and the risk of Alzheimer's disease in late onset families. [see comment]. Science 261: 921-923.

Dahlman I, Eaves IA, Kosoy R, Morrison VA, Heward J, Gough SC et al (2002). Parameters for reliable results in genetic association studies in common disease. Nat Genet 30: 149-150.

DREAM Trial Investigators Gerstein HC, Yusuf S, Bosch J, Pogue J, Sheridan P, Dinccag $\mathrm{N}$ et al (2006). Effect of rosiglitazone on the frequency of diabetes in patients with impaired glucose tolerance or impaired fasting glucose: a randomised controlled trial. [see comment] [erratum appears in lancet. 2006 nov 18;368(9549):1770]. Lancet 368: 1096-1105.

Dreses-Werringloer $\mathrm{U}$, Lambert JC, Vingtdeux V, Zhao $\mathrm{H}$, Vais $\mathrm{H}$, Siebert A et al (2008). A polymorphism in calhm1 influences ca2+ homeostasis, abeta levels, and Alzheimer's disease risk. Cell 133: 1149-1161.

Dunys J, Kawarai T, Giaime E, Wilk S, Herrant M, Auberger P et al (2007). Study on the putative contribution of caspases and the proteasome to the degradation of aph-1a and pen-2. Neurodegener Dis 4: 156-163.

E15 definitions for genomic biomarkers, pharmacogenomics, pharmacogenetics, genomic data and sample coding categories (2008). Http://www.Fda.Gov/cber/ gdlns/iche15term. Htm.

Epstein RA (2007). Conflicts of interest in health care: who guards the guardians? Perspect Biol Med 50: 72-88.

Fovenyi J (2006). [Experiences of the dream trial]. Orvosi Hetilap 147: 2523-2526.

Frayling TM (2007). Genome-wide association studies provide new insights into type 2 diabetes aetiology. Nat Rev Genet 8: 657-662.

Gerstein HC, Yusuf S, Holman R, Bosch J, Pogue J, The DTI (2004). Rationale, design and recruitment characteristics of a large, simple international trial of diabetes prevention: the dream trial. Diabetologia 47: 1519-1527.

Goate A, Chartier-Harlin MC, Mullan M, Brown J, Crawford F, Fidani L et al (1991). Segregation of a missense mutation in the amyloid precursor protein gene with familial Alzheimer's disease. [see comment]. Nature 349: 704-706.

Goldberg RB (2007). The new clinical trials with thiazolidinediones - dream, adopt and Chicago: promises fulfilled? Curr Opin Lipidol 18: 435-442.

Goldgaber D, Lerman MI, McBride OW, Saffiotti U, Gajdusek DC (1987). Characterization and chromosomal localization of a cdna encoding brain amyloid of Alzheimer's disease. Science 235: 877-880.

Goldstein DB, Tate SK, Sisodiya SM (2003). Pharmacogenetics goes genomic [erratum appears in nat rev genet. 2004 jan;5(1):76]. Nat Rev Genet 4: 937-947.

Grant SF, Thorleifsson G, Reynisdottir I, Benediktsson R, Manolescu A, Sainz J et al (2006). Variant of transcription factor 7 -like 2 (tcf7l2) gene confers risk of type 2 diabetes. Nat Genet 38: 320-323.
Harris FM, Brecht WJ, Xu Q, Tesseur I, Kekonius L, Wyss-Coray T et al (2003). Carboxyl-terminal-truncated apolipoprotein e4 causes Alzheimer's disease-like neurodegeneration and behavioral deficits in transgenic mice. Proc Natl Acad Sci USA 100: 10966-10971.

Helgason A, Palsson S, Thorleifsson G, Grant SF, Emilsson V, Gunnarsdottir S et al (2007). Refining the impact of tcf7l2 gene variants on type 2 diabetes and adaptive evolution. Nat Genet 39: 218-225

Hetherington S, Hughes AR, Mosteller M, Shortino D, Baker KL, Spreen W et al (2002). Genetic variations in hla-b region and hypersensitivity reactions to Abacavir. Lancet 359: 1121-1122.

Hetherington S, McGuirk S, Powell G, Cutrell A, Naderer O, Spreen B et al (2001). Hypersensitivity reactions during therapy with the nucleoside reverse transcriptase inhibitor abacavir. Clin Ther 23: 1603-1614.

Hirschhorn JN, Lohmueller K, Byrne E, Hirschhorn K (2002). A comprehensive review of genetic association studies. Genet Med 4: 45-61.

Hisatomi T, Nakazawa T, Noda K, Almulki L, Miyahara S, Nakao S et al (2008). Hiv protease inhibitors provide neuroprotection through inhibition of mitochondrial apoptosis in mice. J Clin Invest 118: 2025-2038.

Hughes AR, Spreen WR, Mosteller M, Warren LL, Lai EH, Brothers CH et al (2008). Pharmacogenetics of hypersensitivity to abacavir: Pgx hypothesis to confirmation to clinical utility? Pharmacogenomics J, advance online publication, March 11, 2008; doi:10.1038/tpj.2008.3

Hunter DJ, Kraft P, Jacobs KB, Cox DG, Yeager M, Hankinson SE et al (2007). A genome-wide association study identifies alleles in fgfr2 associated with risk of sporadic postmenopausal breast cancer. Nat Genet 39: 870-874.

loannidis JP (2007). Non-replication and inconsistency in the genome-wide association setting. Hum Hered 64: 203-213.

Jani-Acsadi A, Krajewski K, Shy ME (2008). Charcot-marie-tooth neuropathies: diagnosis and management. Semin Neurol 28: 185-194.

Kamino K, Sato S, Sakaki Y, Yoshiiwa A, Nishiwaki Y, Takeda M et al (1996). Three different mutations of presenilin 1 gene in early-onset Alzheimer's disease families. Neurosci Lett 208: 195-198.

Koeppen $\mathrm{AH}$ (2005). The pathogenesis of spinocerebellar ataxia. Cerebellum 4 62-73.

Lago RM, Singh PP, Nesto RW (2007). Congestive heart failure and cardiovascular death in patients with prediabetes and type 2 diabetes given thiazolidinediones: a meta-analysis of randomised clinical trials. [see comment]. Lancet 370: $1129-1136$

Lai E, Riley J, Purvis I, Roses A (1998). A 4-mb high-density single nucleotide polymorphism-based map around human apoe. Genomics 54: 31-38

Lee JH, Cheng R, Schupf N, Manly J, Lantigua R, Stern Y et al (2007). The association between genetic variants in sorl1 and Alzheimer disease in an urban multiethnic, community-based cohort. Arch Neurol 64: 501-506.

Lesko LJ (2007). Paving the critical path: how can clinical pharmacology help achieve the vision? Clin Pharmacol Ther 81: 170-177.

Li H, Wetten S, Li L, St Jean PL, Upmanyu R, Surh L et al (2008). Candidate singlenucleotide polymorphisms from a genomewide association study of Alzheimer disease. Arch Neurol 65: 45-53.

Libioulle C, Louis E, Hansoul S, Sandor C, Farnir F, Franchimont D et al (2007). Novel Crohn's disease locus identified by genome-wide association maps to a gene desert on $5 \mathrm{p} 13.1$ and modulates expression of ptger4. PLoS Genet 3: e58.

Mahley RW, Weisgraber KH, Huang Y (2006). Apolipoprotein e4: a causative factor and therapeutic target in neuropathology, including alzheimer's disease. [see comment]. Proc Natl Acad Sci USA 103: 5644-5651.

Malhotra AK, Murphy Jr GM, Kennedy JL (2004). Pharmacogenetics of psychotropic drug response. Am J Psychiatry 161: 780-796.

Mallal S (2007). Predict-1: A novel randomised prospective study to determine the clinical utility of HLA-B ${ }^{\star} 5701$ screening to reduce abacavir hypersensitivity in hivinfected subjects (study cna106030): 4th IAS Conference on HIV Pathogenesis, Treatment and Prevention: Late Breaker Track B. Sydney, Australia.

Mallal S (2008). The predict-1 study: a randomized, double-blind trial to determine the clinical utility of HLA-B 5701 pharmacogenetic screening for abacavir hypersensitivity in hiv-infected patients (study cna106030). New Eng J Med 358 568-579.

Mallal S, Nolan D, Witt C, Masel G, Martin AM, Moore C et al (2002). Association between presence of HLA-B ${ }^{\star} 5701$, hla-dr7, and hla-dq3 and hypersensitivity to hiv-1 reverse-transcriptase inhibitor abacavir. [see comment]. Lancet 359 727-732.

Maron BJ, Hauser RG (2007). Perspectives on the failure of pharmaceutical and medical device industries to fully protect public health interests. Am J Cardiol 100: $147-151$.

Martin ER, Lai EH, Gilbert JR, Rogala AR, Afshari AJ, Riley J et al (2000). Snping away at complex diseases: analysis of single-nucleotide polymorphisms around apoe in Alzheimer disease. Am J Hum Genet 67: 383-394. 
McCarthy MI, Abecasis GR, Cardon LR, Goldstein DB, Little J, loannidis JP et al (2008). Genome-wide association studies for complex traits: consensus, uncertainty and challenges. Nat Rev Genet 9: 356-369.

McKusick VA (2006). A 60-year tale of spots, maps, and genes. Annu Rev Genomics Hum Genet 7: 1-27.

McKusick VA (2007). Mendelian inheritance in man and its online version, omim. Am $J$ Hum Genet 80: 588-604.

Meng Y, Lee JH, Cheng R, St George-Hyslop P, Mayeux R, Farrer LA et al (2007). Association between sorl1 and Alzheimer's disease in a genome-wide study. Neuroreport 18: 1761-1764.

Need AC, Motulsky AG, Goldstein DB (2005). Priorities and standards in pharmacogenetic research. Nat Genet 37: 671-681.

Nissen SE, Wolski K (2007). Effect of rosiglitazone on the risk of myocardial infarction and death from cardiovascular causes. [see comment] [erratum appears in $\mathrm{n}$ engl $\mathrm{j}$ med. 2007 jul 5;357(1):100.]. New Engl J Med 356: 2457-2471.

North BV, Curtis D, Martin ER, Lai EH, Roses AD, Sham PC et al (2004). Further investigation of linkage disequilibrium snps and their ability to identify associated susceptibility loci. Ann Hum Genet 68: 240-248.

Okura Y, Matsumoto $Y$ (2008). DNA vaccine therapy for Alzheimer's disease: present status and future direction. Rejuvenation Res 11: 301-308.

Orr MS, Goodsaid F, Amur S, Rudman A, Frueh FW (2007). The experience with voluntary genomic data submissions at the fda and a vision for the future of the voluntary data submission program. Clin Pharmacol Ther 81: 294-297.

Passamonti L, Muglia M, Magariello A, Bellesi M, Conforti FL, Mazzei R et al (2004). Further evidence of genetic heterogeneity in autosomal dominant distal motor neuronopathy. Neuromuscul Disord 14: 705-710.

Peck C (2007). Preventing postmarketing changes in recommended doses and marketing withdrawals. Ernst Schering Res Found Workshop 59: 209-216.

Phillips EJ, Mallal SA (2008). Pharmacogenetics and the potential for the individualization of antiretroviral therapy. Curr Opin Infect Dis 21: 16-24.

Pride M, Seubert P, Grundman M, Hagen M, Eldridge J, Black RS et al (2008). Progress in the active immunotherapeutic approach to Alzheimer's disease: clinical investigations into an1792-associated meningoencephalitis. Neurodegener Dis 5: 194-196.

Raelson JV, Little RD, Ruether A, Fournier H, Paquin B, Van Eerdewegh P et al (2007). Genome-wide association study for Crohn's disease in the Quebec founder population identifies multiple validated disease loci. Proc Natl Acad Sci USA 104: 14747-14752

Reichert JM (2003). Trends in development and approval times for new therapeutics in the United States. Nat Rev Drug Discov 2: 695-702.

Risner ME, Saunders AM, Altman JF, Ormandy GC, Craft S, Foley IM, et al, Rosiglitazone in Alzheimer's Disease Study G (2006). Efficacy of rosiglitazone in a genetically defined population with mild-to-moderate Alzheimer's disease* ${ }^{\star}$ see comment]. Pharmacogenomics J 6: 246-254.

Roses A (2008a). The medical and pharmaco-economic roles of pharmacogenetics: Alzheimer's disease as a model of efficacy and HLA-B*5701 as a model of safety. Neuropsychopharmacol Rev. CNS Drug Discovery \& Development: Challenges and Opportunities (in press).

Roses A (2008b). Stimulation of cholecystokinin-a receptors with gl181771x: a failed clinical trial that did not test the proposed mechanism of action. Clin Pharmacol Ther (in press).

Roses AD (1996). From genes to mechanisms to therapies: lessons to be learned from neurological disorders. [see comment]. Nat Med 2: 267-269.

Roses AD (1997). A model for susceptibility polymorphisms for complex diseases: apolipoprotein e and alzheimer disease. Neurogenetics 1: 3-11.

Roses AD (2004). Pharmacogenetics and drug development: the path to safer and more effective drugs. Nat Rev Genet 5: 645-656.

Roses AD (2007). Genome-wide screening for drug discovery and companion diagnostics. Expert Opin Drug Discov 2: 489-501.

Roses AD (2008c). Pharmacogenetics in drug discovery and development: a translational perspective. Nat Rev Drug Discov (in press).

Roses AD, Gilbert J, Xu PT, Sullivan P, Popko B, Burkhart DS et al (1998). Cis-acting human apoe tissue expression element is associated with human pattern of intraneuronal apoe in transgenic mice. Neurobiol Aging 19: S53-S58.

Roses AD, Saunders AM, Huang Y, Strum J, Weisgraber KH, Mahley RW (2007a). Complex disease-associated pharmacogenetics: drug efficacy, drug safety, and confirmation of a pathogenetic hypothesis (Alzheimer's disease). Pharmacogenomics J 7: 10-28.
Roses AD, St Jean PL, Ehm MG (2007b). Use of whole-genome association scans in disease gene identification, drug discovery and development. Idrugs 10 797-804.

Russman BS (2007). Spinal muscular atrophy: clinical classification and disease heterogeneity. J Child Neurol 22: 946-951.

Saunders AM, Roses AD (1993). Apolipoprotein e4 allele frequency, ischemic cerebrovascular disease, and Alzheimer's disease. [see comment]. Stroke 24 1416-1417.

Schellenberg GD, Bird TD, Wijsman EM, Orr HT, Anderson L, Nemens E et al (1992). Genetic linkage evidence for a familial Alzheimer's disease locus on chromosome 14. Science 258: 668-671.

Schmechel DE, Goldgaber D, Burkhart DS, Gilbert JR, Gajdusek DC, Roses AD et al (1988). Cellular localization of messenger rna encoding amyloidbeta-protein in normal tissue and in alzheimer disease. Alzheimer Dis Assoc Disord 2: 96-111.

Schymick JC, Scholz SW, Fung HC, Britton A, Arepalli S, Gibbs JR et al (2007). Genome-wide genotyping in amyotrophic lateral sclerosis and neurologically normal controls: first stage analysis and public release of data. [see comment] Lancet Neurol 6: 322-328.

Scott LJ, Mohlke KL, Bonnycastle LL, Willer CJ, Li Y, Duren WL et al (2007). A genome-wide association study of type 2 diabetes in Finns detects multiple susceptibility variants. Science 316: 1341-1345.

Selkoe DJ, Wolfe MS (2007). Presenilin: running with scissors in the membrane. Cell 131: 215-221.

Serretti A, Kato M, Kennedy JL (2008). Pharmacogenetic studies in depression: a proposal for methodologic guidelines. Pharmacogenomics J 8: 90-100.

Sherrington R, Froelich S, Sorbi S, Campion D, Chi H, Rogaeva EA et al (1996). Alzheimer's disease associated with mutations in presenilin 2 is rare and variably penetrant. Hum Mol Genet 5: 985-988.

Sladek R, Rocheleau G, Rung J, Dina C, Shen L, Serre D et al (2007). A genomewide association study identifies novel risk loci for type 2 diabetes. [see comment]. Nature 445: 881-885

St George-Hyslop PH, Tanzi RE, Polinsky RJ, Haines JL, Nee L, Watkins PC et al (1987). The genetic defect causing familial Alzheimer's disease maps on chromosome 21. Science 235: 885-890.

Strittmatter WJ, Huang DY, Bhasin R, Roses AD, Goldgaber D (1993). Avid binding of beta an amyloid peptide to its own precursor. Exp Neurol 122: 327-334.

Tanzi RE, Gusella JF, Watkins PC, Bruns GA, St George-Hyslop P, Van Keuren ML et al (1987). Amyloid beta protein gene: Cdna, mrna distribution, and genetic linkage near the Alzheimer locus. Science 235: 880-884.

Townsend M, Mehta T, Selkoe DJ (2007). Soluble abeta inhibits specific signal transduction cascades common to the insulin receptor pathway. $J$ Biol Chem 282: 33305-33312.

Walsh DM, Selkoe DJ (2007). A beta oligomers-a decade of discovery. J Neurochem 101: 1172-1184.

Warren LL, Hughes AR, Lai EH, Zaykin DV, Haneline SA, Bansal AT, et al, teams CNAaCs (2007). Use of pairwise marker combination and recursive partitioning in a pharmacogenetic genome-wide scan. Pharmacogenomics $J$ 7: $180-189$.

Wellcome Trust Case Control Consortium (2007). Genome-wide association study of 14,000 cases of seven common diseases and 3,000 shared controls. [see comment]. Nature 447: 661-678.

Xu Q, Bernardo A, Walker D, Kanegawa T, Mahley RW, Huang Y et al (2006). Profile and regulation of apolipoprotein e (apoe) expression in the cns in mice with targeting of green fluorescent protein gene to the apoe locus. J Neurosci 26 4985-4994.

Zaks TZ, Akkari A, Briley L, Mosteler M, Stead AG, Koch KM et al (2006). Role of pharmacogenetic studies in early clinical development: phase i studies with lapatinib. J Clin Oncol 24: 3029.

Zanke BW, Greenwood CM, Rangrej J, Kustra R, Tenesa A, Farrington SM et al (2007). Genome-wide association scan identifies a colorectal cancer susceptibility locus on chromosome 8q24. Nat Genet 39: 989-994.

Zeggini E, Weedon MN, Lindgren CM, Frayling TM, Elliott KS, Lango H et al (2007). Replication of genome-wide association signals in UK samples reveals risk loci for type 2 diabetes. Science 316: 1336-1341.

Ziedan NI, Kadri H, Westwell AD (2008). The development of pro-apoptotic cancer therapeutics. Mini Rev Med Chem 8: 711-718. 